\title{
Leprosy in the new millennium
}

The World Health Organization (WHO) started the decade with the bold slogan of 'eliminating leprosy as public health problem by 2000 '. The WHO initiative has galvanised leprosy control programmes world-wide, but it is now clear that this target will not be achieved. As the millennium approached, the slogan was quietly dropped and many leprosy workers and governments are disappointed. The unique biology of Mycobacterium leprae and its interaction with the human host have meant that the elimination target was unattainable and leprosy will continue to be a global problem for many decades.

The transmission of $M$. leprae is still only partially understood. Lepromatous patients have selective cellmediated anergy against $M$. leprae and the mycobacterium multiplies unchecked in the target organs of skin, peripheral nerve and nasal mucosa. Untreated lepromatous patients discharge abundant organisms in droplets from their nasal mucosa into the environment. $M$. leprae is a hardy organism that, in a South Indian laboratory, has been shown to remain viable for 5 months after drying in the shade [1]. Studies in Indonesia [2] and Ethiopia [3] with PCR primers to detect $M$. leprae DNA in nasal swabs have shown that up to $5 \%$ of the population and $16 \%$ of leprosy workers in these endemic areas carry $M$. leprae DNA in their noses. The combination of an environmentally welladapted organism, high carriage rates and a long incubation period means that, even with effective antibiotics, transmission will continue for a long time and this is reflected in the global trends. New cases occur at a stable rate of c. 600000 each year, with continuing high rates of childhood cases confirming on-going transmission [4]. Intensive leprosy elimination campaigns were held in 1999 with the aim of flushing out remaining cases, perhaps in time for the millennium. What has been surprising about these campaigns is how many new cases have been detected. A weeklong campaign in Nepal found 11696 new cases, thereby doubling the national case load [5].

There are presently 115 leprosy patients on the central leprosy register in the UK and 32 new cases have been treated at the Hospital for Tropical Diseases in the past 4 years [6]. All result from infection abroad. The ethnicity of these leprosy patients has changed: in the 1950 s West Indian patients accounted for $8 \%$ of the annual notifications, but only two West Indian cases have been reported since 1990 . Now almost $50 \%$ of the cases in England and Wales come from the Indian subcontinent. Three of the recent cases seen at the Hospital for Tropical Diseases are Caucasian-British individuals who had spent $8-42$ years overseas. Casual visitors to leprosy endemic areas can be reassured that they are highly unlikely to contract leprosy.

M. leprae still cannot be grown in vitro and the best animal models are the nude mouse footpad and the nine-banded armadillo. These are useful for detecting drug resistance and producing whole preparations of $M$. leprae respectively. The genome for M. leprae has now been almost completely sequenced at the Institut Pasteur in Paris; it is $50 \%$ smaller than that of $M$. tuberculosis. M. leprae has c. 1100 housekeeping genes, 300 genes encoding proteins common to other mycobacteria, 50 for exported proteins and c. 60 for unique proteins. Analysis of this last group of proteins will be critical for developing new diagnostic tests, as well as understanding how $M$. leprae can survive and become dormant [7].

Leprosy remains a clinical diagnosis, resting on the recognition of typical skin lesions together with thickened peripheral nerves and confirmed, in borderline and lepromatous cases, by finding acid-fast bacilli in slit skin smears. Histology of skin or nerve biopsies remains crucial for diagnosis in atypical cases and for classification. Staining nasal mucus for mycobacteria is unhelpful, as it does not confirm the diagnosis and puts laboratory staff at unnecessary risk.

There have been numerous attempts to develop a serological test for diagnosis and screening. So far, none has been found to exhibit sufficient sensitivity or specificity to be clinically useful: while lepromatous patients produce antibodies to many candidate antigens the antibody response in tuberculoid patients is weak or absent. The most promising candidate antibody - that against phenolic glycolipid - is absent in almost $50 \%$ of paucibacillary cases [8]. It was hoped that a PCR for $M$. leprae DNA would prove helpful in diagnosing tuberculoid cases where the bacterial load is low. However, the PCR primers used so far have poor sensitivity in paucibacillary cases and false positive results have also proved a problem $[9,10]$.

The success story of the last decade has been the 
introduction and delivery of multi-drug therapy. The triple drug combination of rifampicin, dapsone and clofazimine was introduced in 1982 to counter the growing problem of secondary and primary dapsone resistance that resulted from use of dapsone monotherapy in the previous three decades. The cure rate is high, there are few side-effects and the relapse rate so far is only $c .0 .5 \%$ annually in multibacillary cases. A key component of the success of multi-drug therapy has been the supervision of the monthly rifampicin and clofazimine. In India, leprosy paramedical workers have supervised patient compliance through drug delivery circuits in towns and villages. The anti-leprosy drugs have been provided to leprosy endemic countries free of charge by the Sasakawa foundation (Tokyo, Japan).

A single-dose triple combination of rifampicin, ofloxacin and minocycline achieved improvement in $98 \%$ of patients with single skin lesions in trials in India [12]. Although the study had major flaws and single-dose treatment is less effective than the conventional 6month treatment for paucibacillary leprosy, this is an operationally attractive regimen and has been recommended for use by the WHO. However, giving singledose treatment to kill an intracellular organism is asking a lot; the drugs act only on metabolically active organisms, so that non-growing or dormant organisms will not be killed [13].

A major controversy is raging over the optimum duration of treatment. The last WHO expert committee recommended that treatment for multibacillary cases could be reduced from 24 to 12 months [14]. Proponents of 12 months treatment argue that the relapse rate is low anyway, and many patients are now being over-treated; the dapsone-clofazimine component of the multi-drug regimen will ensure elimination of any spontaneously occurring rifampicin-resistant mutants [15]. Those who would like to retain longer treatment periods argue that relapse rates in patients with initially high bacterial loads are unacceptably high and that it is these patients who will maintain transmission of leprosy in the community [16]. A WHO sponsored trial comparing the two regimens is in progress, but was started only in 1992 and a 7-10-year follow-up is needed to assess relapse rates. A worrying report from West Africa found that patients with a high initial bacterial load (bacterial index $>4+$ ) treated with a combination of rifampicin, clofazimine and dapsone for 2 years had a relapse rate of $30 \%$ [17]. This has just been replicated in India (Girdhar et al.; personal communication). The dilemma is that leprosy cases are now classified on the basis of the number of lesions and slit skin smears are no longer mandatory because of the poor standard of smears in the field. If skin smears are abandoned then those patients in need of longer treatment courses cannot be identified. These arguments illustrate the difficulty in providing policy decisions when a decade-long wait for sound evidence is needed.
The major challenge for leprosy workers now is the management of the immunological complications of leprosy: nerve damage and reactions. Nerve damage can be acute, chronic or silent and results from intraneural cell-mediated immune activation of $\mathrm{T}$ cells and macrophages against M. leprae antigens [18]. Among multibacillary patients $10-30 \%$ have peripheral nerve damage at the time of presentation. Further nerve damage also occurs during and after treatment. Steroids remain the mainstay of treatment for recent leprosy nerve damage and a pilot study has indicated that prophylactic steroids ( $20 \mathrm{mg}$ daily for 3 months) may decrease the incidence of reactions in multibacillary patients by $50 \%$ [19]. A double-blind randomised controlled trial in India is currently testing this effect. It will be important to determine whether the protective effect is maintained after stopping steroids. Other immunosuppressants are also being tested to see whether drugs such as azathioprine and cyclosporin A have a role in reducing tissue-damaging immune activity. It is estimated that 3 million people globally have disabilities resulting from leprosy. Peripheral nerve damage may be life-long and protecting anaesthetic hands and feet in a resource-poor country is a long-term challenge for patients and doctors.

It was feared that HIV infection might be a risk factor for leprosy with HIV immune paresis causing conversion of subclinical cases and increased numbers of lepromatous cases. However, studies in several countries [20-23] have found that HIV infection is not a risk factor for leprosy. A prospective case-controlled study in Uganda showed a significant association between HIV seropositivity and the development of neuritis and reactions [24]. This was unexpected as CD4+ cells mediate reversal reactions. Brazilian workers have reported on the immunohistology of 11 patients co-infected with HIV and leprosy; all had typical leprosy histology. Even patients with low circulating CD4 counts had normal granuloma formation with abundant CD4 cells [25].

Last year visitors to the WHO leprosy web site found the logo 'A world without leprosy is not a dream'. This may eventually come true, but for now important challenges remain in detecting and treating patients and preventing and managing disability.

DIANA N. J. LOCKWOOD

London School of Hygiene and Tropical Medicine, Keppel St, London WC1E 7HT

(e-mail: diana.lockwood@1shtm.ac.uk)

\section{References}

1. Desikan KV, Sreevasta. Extended studies on the viability of Mycobacterium leprae outside the human body. Lepr Rev 1995; 66: 287-295.

2. Hatta H, van Beers SM, Madjid B, de Wit MYL, Klatser PR. Distribution and persistence of Mycobacterium leprae nasal carriage among a population in which leprosy is endemic in Indonesia. Trans $R$ Soc Trop Med Hyg 1995; 89: 381-385. 
3. Ramaprasad P, Fernando A, Madhale S et al. Transmission and protection in leprosy: indications of the role of mucosal immunity. Lepr Rev 1997; 68: 301-315.

4. World Health Organization. Global case-detection trend in leprosy. Wkly Epidemiol Rec 1997; 72: 173-180.

5. Baral JP, Shwe T. The Nepal nationwide leprosy elimination campaign. Lepr Rev 1999 (in press).

6. Vanbunyen P, Leese J, Lockwood DNJ. Leprosy in England and Wales. Comm Dis and Public Health 1999; 2: 119-121.

7. Roche P, Dockrell H, Brennan P. Progress in research towards a world without leprosy. Report of a WHO meeting in Ethiopia, February 1998. Lepr Rev 1998; 69: 151-159.

8. Smith PG. The serodiagnosis of leprosy. Lepr Rev 1992; 63: 97-100.

9. Wichitwechkarn J, Karnjan S, Shuntawuttisettee S, Sornprasit C, Kamirapap K, Peeralorn S. Detection of Mycobacterium leprae infection by PCR. J Clin Microbiol 1995; 33: 45-49.

10. Jamil S, Keer JT, Lucas SB et al. Use of polymerase chain reaction to assess efficacy of leprosy chemotherapy. Lancet 1993; 342: 264-268.

11. WHO Leprosy Unit. Risk of relapse in leprosy. WHO Document WHO/CTD/LEP 94.1.

12. Single Lesion Multicentre Group Trial Group. Efficacy of single dose multidrug therapy for the treatment of single lesion paucibacillary leprosy. Indian J Lepr 1997; 69: 121-129.

13. Katoch VM. Is there a microbiological rationale for single dose treatment of leprosy? Lepr Rev 1998; 69: 2-5.

14. WHO technical report Series 874. WHO expert committee on leprosy. World Health Organization, Geneva. 1998.

15. Ji B. Why multidrug therapy for multibacillary leprosy can be shortened to twelve months. Lepr Rev 1998; 69: 106-109.

16. Waters M. Commentary. Is it safe to shorten multidrug therapy for lepromatous (LL and BL) leprosy to twelve months? Lepr Rev 1998; 69: 110-111.

17. Jamet P, Ji B and the Marchoux Chemotherapy Study Group. Relapse after long term follow-up of multibacillary patients treated by the WHO multidrug regimen. Int J Lepr 1995; 63: 195-201.

18. Britton WJ. The management of leprosy reversal reactions. Lepr Rev 1998; 69: 225-234.

19. Croft RP, Nicholls P, Anderson A, van Brakel W, Smith WCS, Richardus JH. Effect of prophylactic steroids on the incidence of reactions in newly diagnosed multibacillary leprosy patients. Int J Lepr 1999; 67: 75-77.

20. Ponnighaus JM, Mwanjasi JL, Fine PEM et al. Is HIV infection a risk factor for leprosy? Int J Lepr 1991; 59: 221-228.

21. Kawuma HJS, Bwire R, Adatu-Engwau F. Leprosy and infection with the human immunodeficiency virus in Uganda; a case control study. Int J Lepr 1994; 62: 521-526.

22. Lienhardt C, Kamate B, Jamet P et al. Effect of HIV infection on leprosy: a three-year survey in Bamako, Mali. Int $J$ Lepr Other Mycobact Dis 1996; 64: 383-391.

23. Sekar B, Jayasheela M, Chattopadhya D et al. Prevalence of HIV infection and high-risk characteristics among leprosy patients of south India; a case control study. Int J Lepr 1994; 62: 527.

24. Bwire R, Kawuma HJS. Type 1 reactions in leprosy, neuritis and steroid therapy: the impact of the human immunodeficiency virus. Trans $R$ Soc Trop Med Hyg 1994; 88: 315-316.

25. Sampaio EP, Caneshi JRT, Nery JAC et al. Cellular immune response to Mycobacterium leprae infection in human immunodeficiency virus infected individuals. Infect Immun 1995; 63: $18848-18854$. 